\title{
Design and Implementation of a Level Detector
}

\author{
Mehdi Hassani \\ Dept. of Electrical Engineering \\ University of Malaya \\ me.hassani@gmail.com
}

\author{
Hamed Tebianian \\ Dept. of Electrical Engineering Azad \\ Islamic University of Tehran
}

\author{
Saad Mekhilef \\ Dept. of Electrical Engineering \\ University of Malaya
}

\author{
Amirhossein Mehbodniya \\ Dept. of Electrical Engineering \\ University of Malaya
}

\begin{abstract}
This paper presents the design and implementation of a level detector. Silometer is an electromechanical level detector which can measure the level in silos, tanks, bunkers and any kind of containers, depending on the sensing weight and strain force. The Silometer is mostly applicable in dusty, harsh and coarse environments, but it is also useful to be employed in other environments such as in liquid and so on.
\end{abstract}

\section{INTRODUCTION}

In the past few decades, different kinds of level detectors have been produced and developed. Each kind of these sensors possesses a special operating mechanism. The sensors can generally be categorized into two main groups, labelled as electrical and electromechanical sensors [1], [2]. The electrical level detectors work by applying electrical waves, such as ultrasonic. These kinds of sensors have longer life and require less maintenance as compared to electromechanical sensors. Their complicated signal processing, however, makes them expensive sensors to be used. On the other hand, most of them are prepared for individual environment only and they cannot be useful in dusty, harsh and caustic environments. Electromechanical sensors require more maintenance and have a shorter period of life, particularly in terms of their mechanical nature, but they are very suitable to be employed in harsh environments [3]. At the present time most of factories that are producing or working with dusty products or materials, are facing the problem of measuring their silos, bunkers or containers. Using this technology, factories are able to access and retrieve accurate information about their products. Briefly silometer can address following issues:

- In most silos, still use a very primary method of height measurement such as throwing a band meter through them. Whereas, the operator should use a ladder or highlevel stairs for this purpose; and at first step, this problem is entirely resolved by Silometer installation.

- This apparatus is so simple for operators to work with. Also it shows the last status of silos and draws graphs for each of them. With these graphs the observant can easily check the production status of last working shift.

- $\quad$ It can provide a comprehensive program for monitoring of all governmental silos in the country, based on NET programming. So the total stock in each province or country, at any moment can be check.

- In traditional method, operator often requires to open silo's door, which it may have some hazards such as falling objects, therefore, Silometer installation can increase the safety level.

\section{System Description And Applications}

Silometer is an electromechanical level detector. It sends a sensing weight through a desired environment. When the sensing weight reaches the surface of the silo material, the tension sensor can detect the slackness of the wire and this enables the main processor to calculate the empty height of the silo. Two stepper motors were used in silometer. With attention to the motors which used, it will be obvious that the algorithms of height calculation are based on counting the number of the motor pulses. To further visualize how this system works, the procedure used is explained as follows: When the measure command is sent to the Silometer, the main electro motor, which is coupled to a pulley, starts to turn and send the sensing weight through the environment of the silo. Simultaneously, another stepper motor, which is located at the bottom of the main motor, also starts to turn. The main role of this motor is to regularly collect the wire on the pulley. When the sensing weight reaches the surface of the material in the silo by the mechanism explained above, the main processor calculates the empty height of the silo. At the same time, the main motor also begins to collect the wire and pull the sensing weight up. Thus, it is rather obvious that the main processor controls both the rolling and unrolling processes of the wire and that it rolls the wire, as much as the pulses number which are initially unrolled. It is important to highlight that when the 
Silometer does not work, the weight must be withstood using another part, because putting the weight on motor shaft all the time, could cause damages to the main motor after long time. For this reason, an electromechanically lock was designed for the Silometer and its role is to withstand the moving system weight during rest time. The tension sensor was designed in such a way that it is very simple. It consists of two ball bearings which are fixed against each other, and both are joined to two springs. These ball bearings can only move in their pre-defined groove. When the sensing weight reaches the surface of the material, the wire becomes slack and the two ball bearings are pulled by the springs to touch two different mechanical micro switches. This briefly explains the procedure involved in the Silometer which makes up the first model. However, after the testing, some problems were found and these led to the development of the initial model.

\section{System Structure}

In this section, the two separable parts of the Silometer are explained. First, the electrical part as well as its different blocks is presented and this is followed by a description of the mechanical part of the system and all its components.

\section{A. Electrical part}

The schematic design of the electrical part is shown in Fig. 1 below.

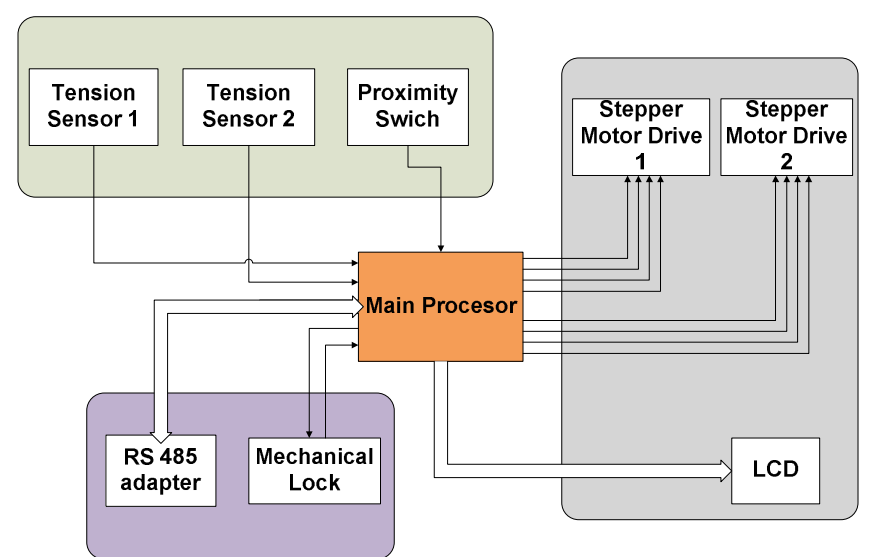

Figure 1. The schematic design of the electrical system

The electrical part shown in Fig. 1 consists of the following blocks:

\section{1) Main processor}

The main processor of the Silometer is one of the powerful ST Microelectronic Co. products, which is known as $\mu$ PSD321X
[4]. It is based on 8051 core and is comprised of many features including extra I/O ports, extra serial port, PWM, huge main flash and an internal SRAM, etc.

\section{2) RS485 adaptor}

Due to the fact that it was supposed to be an industrial instrument, this adapter is needed to support industrial serial connection. The Silometer physical layer for a serial connection is based on RS485 standard [5], [6]. Meanwhile, the IC employed in this part is the ADM485.

\section{3) Motor drives}

Two separated motor drives were designed for two stepper motor. The main stepper motor which acts as the motive force of the Silometer has 6 amperes drive, while the other stepper motor, i.e. for regular rolling of the wire, has 2 amperes drive. For this section, both L297 and L298 7] were used as ICs.

\section{4) Inputs and Outputs}

The inputs and outputs of Silometer are as follows:

Micro switches which consist of tension sensor, electro mechanical lock and regular roller.

Proximity switch which is used for sensing weight calibration. LCD port.

\section{B. Mechanical part}

\section{1) Stepper motors}

A Silometer has two stepper motors with different power. The main stepper motor which has a $13 \mathrm{~kg} / \mathrm{cm}$ rated torque provides the main motive force, whereas the other stepper motor, with $3 \mathrm{~kg} / \mathrm{cm}$ rated torque, supports the linear motion of the regular roller.

\section{2) Tension sensor}

The tension sensor is a mechanical package. It consists of two ball bearings which are connected to two micro switches, and one of them is reserved for the main switch.

\section{3) Main holder}

This part was specifically designed for holding motors, pulley, main shaft, mechanical lock, tension sensor and regular roller. The main holder is illustrated in Fig. 2. 


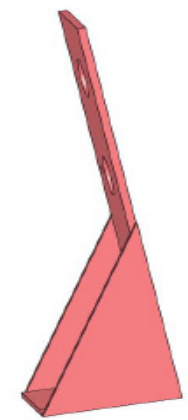

Figure 2. Main holder

\section{4) Regular roller}

It is necessary to roll and unroll the wire regularly to increase the accuracy of the height measurement. This particular part contains two components, namely stepper motor and wounded screw. Fig. 3 presents this part of the system. Based on the figure below, it is obvious that the wounded screw is coupled with stepper motor shaft which converts the rotary motion of the motor to a linear motion. In this way and when the correct algorithm of motor controlling is applied, the rolling process can be controlled and the wire is regularly rolled.

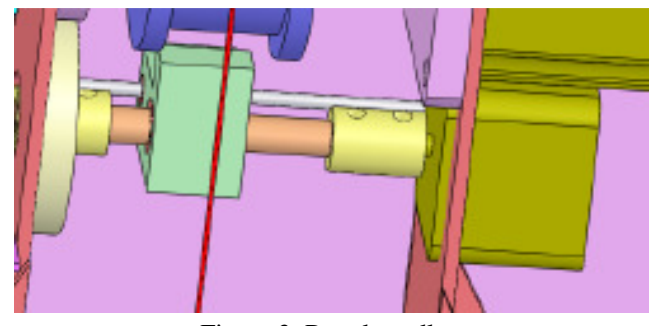

Figure 3. Regular roller

\section{5) Mechanical lock}

The mechanical lock was designed to withstand the whole inertia of the system at the rest time. It includes a solenoid, a stainless steel arm and a spring. The mechanical lock and its components are depicted in Fig. 4 below.

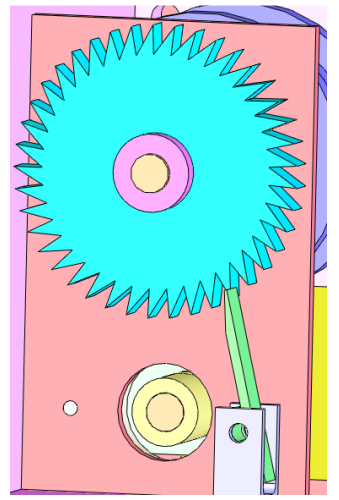

Figure 4. Mechanical lock

\section{6) Main package and holder cylinder}

In general, Silometer comprises two sections labelled as the main case (consisting of all the components) and the cylindrical holder (holding the whole system) which has a proximity switch on its wall. The proximity switch is used in calibrating the sensing weight. In fact, it is referred to and used as a home position (zero position) sensor. Fig. 5 illustrates this particular part of the system.

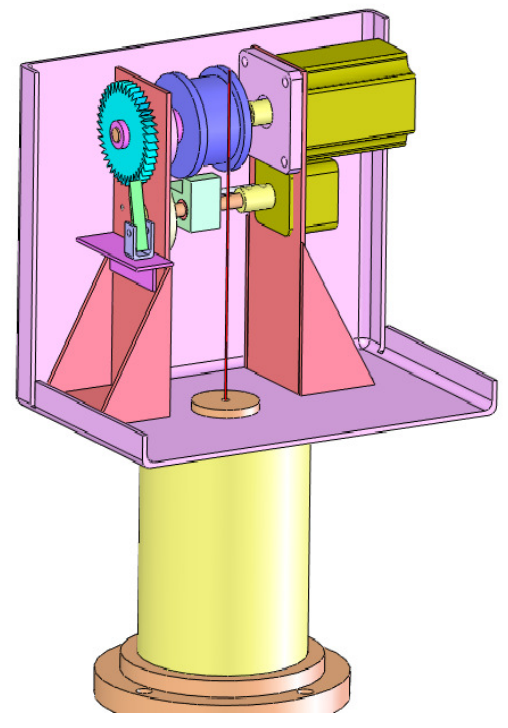

Figure 5. Main package and holder cylinder

\section{Networking And Monotoring}

The ability of this system to connect with the other devices has encouraged its usage as a special instrument in an industrial application. Today, almost all industrial sensors and instruments possess the ability to support different transmitting and receiving standards of data. One of the bus based standard is the RS485 protocol. Since, RS485 bus configuration requires less wiring and also it is reliable against the noise, so the RS485 port was considered for Silometer to achieve a complete sensor as an industrial application. In this way, a network of Silometers is available for any applications which may require more than 2 Silometers. In addition, the bus controller was designed to control the transmission and receipt of data. This bus controller is a RS232 to RS485 converter and it provides the capability of data transmission to computer. The software used in monitoring of the data of the Silometer was also designed in the LabVIEW environment 
[8], [9], [10]. This software has different abilities which include online visual monitoring of silo's height, presenting the height values in different tables, documentation and categorizing information (in daily format, monthly format, etc.), showing data in the form of graphs, highlighting probable errors and many other interesting features (only two pages of this program are presented in Fig. 6 and Fig. 7 respectively).

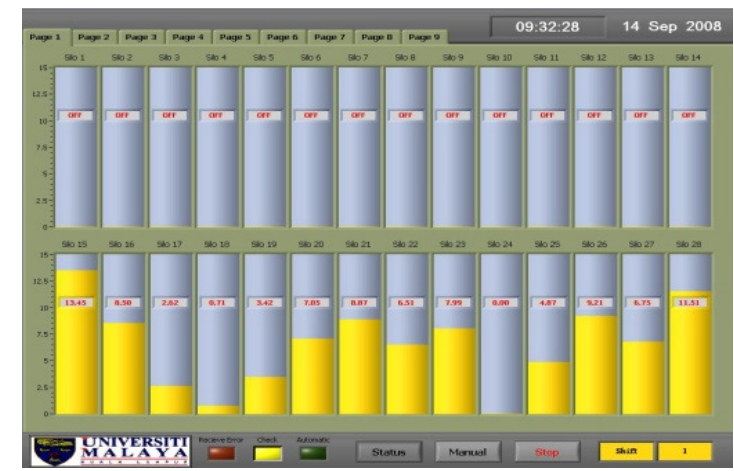

Figure 6. Monitoring Software

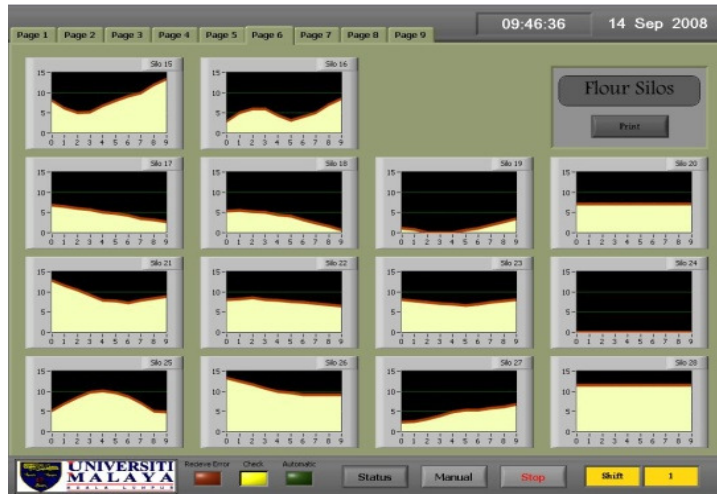

Figure 7. Graph Outputs

V. Testing

The first prototype designed was tested for one year, so some problems were detected in both the electrical and mechanical parts. This first model is shown in Fig. 8.

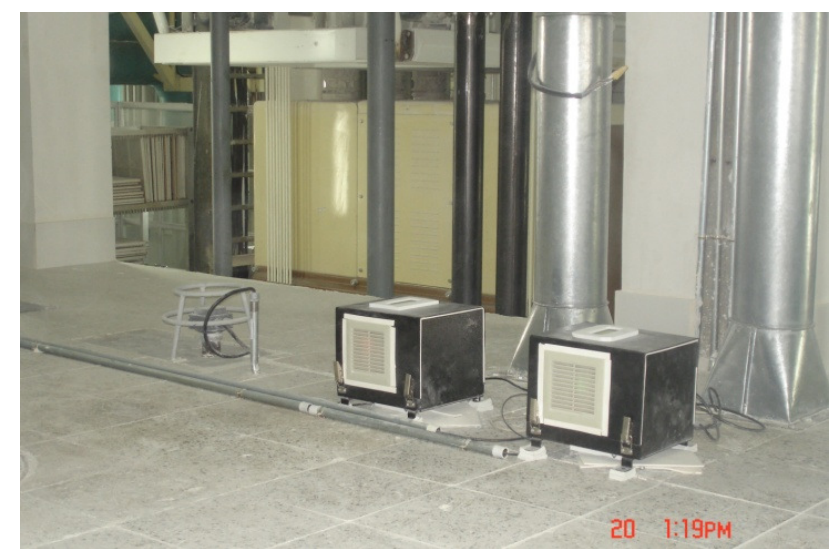

Figure 8 . The first model of Silometer in a flour factory

The latest model was designed based on several studies which were carried out to improve all parts of the system. The model was tested for 9 months and no error or problem has been detected. The latest model is shown in Fig 9.

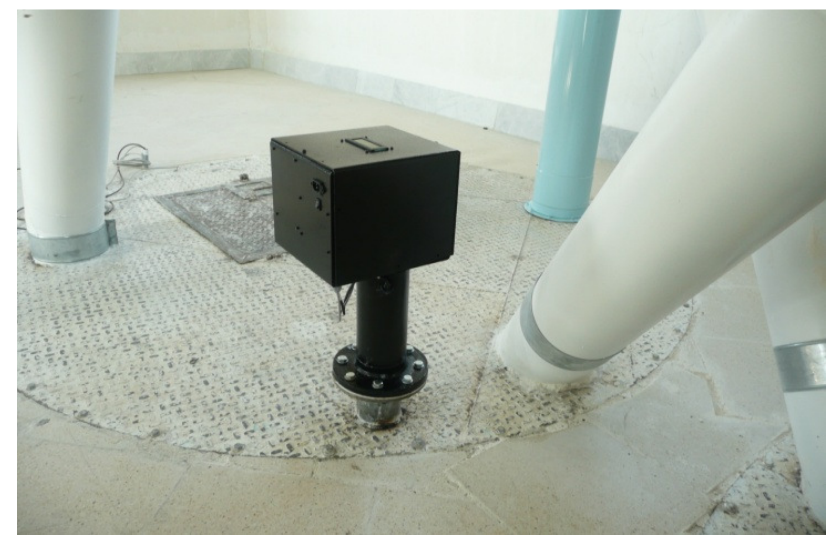

Figure 9. The latest model of the Silometer

\section{Conclusion}

In this paper, design and implementation of a level detector is presented. In this regard the most precious and valuable achievement after three years effort is; manufacturing the prototype. This prototype has been passed different testing situations and it has proved a great reliability and durability as its properties. Consequently it leads to remedy one of the significant difficulties in some industries. Some other merits of this instrument are summarized as follows:

\section{1) monitoring software:}

Silometer is equipped with monitoring software. This has made it simple and interesting for operators to use. In addition, it shows the previous status of the silos and draws graphs (shown in Fig. 9) for each of them. Using these graphs, the 
supervisor can easily monitor or check the production attitude of the previous working shift.

2) Eliminate operators and utilizing automation:

In most silos, it is still common to make use of a primary method of height measurement such as throwing a band meter. In these ways, an operator should use a ladder or high-level stairs to reach himself to top of silos. This problem has entirely been resolved by installing a Silometer.

3) Increase safety level:

In the traditional method, an operator is often required to open a silo's door in order to measure the height. This may impose some hazards such as falling objects, and thus, installing a Silometer can increase the level of safety at a workplace.

\section{REFRENCES}

[1] John G. Webster, "The Measurement, Instrumentation and Sensors handbook"

[2] David M. Scott, "Industrial Process Sensors"

[3] IDC Technology, "Practical Instrumentation for Automation and Process Control"

[4] $\mu$ PSD321X, Data sheet, ST Ltd., 2003

[5] RS-485 and RS-232 Physical Topologies, Sipex, 2006

[6] RS-422 and RS-485 Application Note, " $B \& B$ electronics Manufacturing Company”, 2006.

[7] Stepper Motor System Basics, AMS Company, 2000.

[8] Beyon, J. Y. "LabVIEW Programming, Data Acquisition and Analysis". Upper Saddle River, NJ: Prentice Hall PTR.2001

[9] Wells, L.K. and Jeffrey, T. "LabVIEW for Everyone: Graphical Programming Made Even Easier”. Upper Saddle River, NJ: Prentice Hall PTR.1996

[10] Bishop R. H. “LabVIEW Student Edition 6i”. Upper Saddle River: NJ: Prentice Hall PTR.2001 